\title{
The neutron guide upgrade of the TOSCA spectrometer
}

\author{
Roberto S. Pinna, Svemir Rudic, Stewart F. Parker, Jeff \\ Armstrong, Matteo Zanetti, Goran Škoro, Simon P. Waller, Daniel \\ Zacek, Clive A. Smith, Matthew J. Capstick, \\ David J. McPhail, Daniel E. Pooley, Gareth D. Howells, \\ Giuseppe Gorini and Felix Fernandez-Alonso
}

\section{Published version information}

Citation: RS Pinna et al. "The neutron guide upgrade of the TOSCA spectrometer." Nuclear Instruments and Methods in Physics Research Section A: Accelerators, Spectrometers, Detectors and Associated Equipment, vol. 896 (2018): 68-74.

DOI: 10.1016/i.nima.2018.04.009

(C2018. This manuscript version is made available under the CC-BY-NC-ND 4.0 Licence.

This version is made available in accordance with publisher policies. Please cite only the published version using the reference above. This is the citation assigned by the publisher at the time of issuing the AAM. Please check the publisher's website for any updates. 


\section{Accepted Manuscript}

The neutron guide upgrade of the TOSCA spectrometer

Roberto S. Pinna, Svemir Rudić, Stewart F. Parker, Jeff Armstrong, Matteo Zanetti, Goran Škoro, Simon P. Waller, Daniel Zacek, Clive A. Smith, Matthew J. Capstick, David J. McPhail, Daniel E. Pooley, Gareth D. Howells, Giuseppe Gorini, Felix Fernandez-Alonso

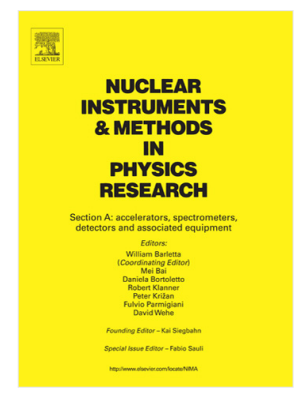

PII: $\quad$ S0168-9002(18)30483-2

DOI: $\quad$ https://doi.org/10.1016/j.nima.2018.04.009

Reference: $\quad$ NIMA 60729

To appear in: $\quad$ Nuclear Inst. and Methods in Physics Research, A

Received date: 28 November 2017

Revised date: 22 March 2018

Accepted date : 5 April 2018

Please cite this article as: R.S. Pinna, S. Rudić, S.F. Parker, J. Armstrong, M. Zanetti, G. Škoro, S.P. Waller, D. Zacek, C.A. Smith, M.J. Capstick, D.J. McPhail, D.E. Pooley, G.D. Howells, G. Gorini, F. Fernandez-Alonso, The neutron guide upgrade of the TOSCA spectrometer, Nuclear Inst. and Methods in Physics Research, A (2018), https://doi.org/10.1016/j.nima.2018.04.009

This is a PDF file of an unedited manuscript that has been accepted for publication. As a service to our customers we are providing this early version of the manuscript. The manuscript will undergo copyediting, typesetting, and review of the resulting proof before it is published in its final form. Please note that during the production process errors may be discovered which could affect the content, and all legal disclaimers that apply to the journal pertain. 


\section{The neutron guide upgrade of the TOSCA spectrometer}

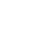

Roberto S. Pinna, ${ }^{1,2}$ Svemir Rudić, ${ }^{1, *}$ Stewart F. Parker, ${ }^{1}$ Jeff Armstrong, ${ }^{1}$ Matteo Zanetti, ${ }^{1,2}$ Goran Škoro, ${ }^{1}$ Simon P. Waller, ${ }^{1}$ Daniel Zacek, ${ }^{1}$ Clive A. Smith, ${ }^{1}$ Matthew J. Capstick, ${ }^{1}$ David J. McPhail, ${ }^{1}$ Daniel E. Pooley, ${ }^{1}$ Gareth D. Howells, ${ }^{1}$ Giuseppe Gorini, ${ }^{2}$ and Felix Fernandez-Alonso ${ }^{1,3}$

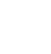

1. ISIS Facility, STFC, Rutherford Appleton Laboratory, Chilton, Didcot OX11 0QX, UK.

2. CNISM, Universita' degli Studi di Milano-Bicocca, Piazza della Scienza 3, 20126 Milano, Italy.

3. Department of Physics and Astronomy, University College London, Gower Street, London WC1E 6BT, UK.

* Author for correspondence.

Contact information:

ISIS Facility

STFC Rutherford Appleton Laboratory

Chilton

Didcot OX11 0QX

UK

Tel: + $44(0) 1235446130$

Fax: + $44(0) 1235445720$

e-mail: svemir.rudic@stfc.ac.uk 


\section{$1 \quad$ Abstract}

3 The primary flightpath of the TOSCA indirect geometry neutron spectrometer has been 4 upgraded with a high- $m 14.636 \mathrm{~m}$ (including $0.418 \mathrm{~m}$ of air gaps) neutron guide composed of 5 ten sections in order to boost the neutron flux at the sample position. The upgraded incident neutron beam has been characterised with the help of the time-of-flight neutron monitor; the beam profile and the gain in the neutron flux data are presented. At an average proton current-on-target of $160 \mu \mathrm{A}$ and proton energy of $800 \mathrm{MeV}$ (ISIS Target Station 1; at the time of the measurements) we have found that the wavelength-integrated neutron flux (from 0.28 $\AA$ to $4.65 \AA$ ) at the position of the TOSCA instrument sample (spatially averaged across a 3.0 $\mathrm{x} 3.0 \mathrm{~cm}^{2}$ surface centred around the $(0,0)$ position) is approximately $2.11 \times 10^{7}$ neutrons $\mathrm{cm}^{-2}$ $\mathrm{s}^{-1}$ while the gain in the neutron flux is as much as 46-fold for neutrons with a wavelength of $2.5 \AA$ A. The instrument's excellent spectral resolution and low spectral background have been preserved upon the upgrade. The much improved count rate allows faster measurements where useful data of hydrogen rich samples can be recorded within minutes, as well as experiments involving smaller samples that were not possible in the past.

Keywords: TOSCA, neutron guide, neutron beam profile, neutron detector, scintillator detector, neutron flux, vibrational spectrometer 
1 1. Introduction

3 TOSCA is an indirect-geometry inelastic neutron spectrometer optimised for high resolution

4 vibrational spectroscopy in the energy transfer region between -24 and $4000 \mathrm{~cm}^{-1}[1,2,3]$. The

5 instrument has been operational for almost two decades and during that time has set the standard for broadband chemical spectroscopy with neutrons [4]. In autumn 2013 as part of

7 the international beamline review [5] it was concluded that for TOSCA to be able to participate in strategic research areas such as $\mathrm{CO}_{2}$ capture and charge storage [6], an increase in the incident neutron flux via the provision of a neutron guide (as opposed to the simple collimation tubes present at the time) would be highly beneficial. Such a development would allow detailed studies of industrially relevant systems containing weak neutron scatters $\left(\mathrm{SO}_{2}\right.$, $\mathrm{CO}, \mathrm{NO})$ as well as faster parametric studies, particularly for hydrogen containing molecules such as hydrocarbons. Additionally, as neutron scattering is an inherently intensity limited technique studies of smaller samples which are too expensive to produce in larger quantities would be possible. Since then, this major upgrade has been implemented which has involved extensive simulations together with the complete redesign of the TOSCA primary spectrometer to house a state-of-the-art, high- $m$ neutron guide and associated chopper system to boost the incident flux on the sample.

Neutron guides are essential in order to boost the neutron flux at a long distance from the neutron source. As neutrons fly nearly parallel to the guide surface they are retained within the tube by a process of external reflection. Traditionally, neutron guides are square or rectangular cross-section tubes made from optically flat materials, usually glass, that has been metal coated with alternating layers of metal with different scattering length densities. More recently, and in particular in case of the longer neutron guides their shape can be rather complex (e.g. elliptical) in order to avoid the loss due to a large number of reflections. Over the years, progress in guide manufacture has led to supermirror coated guides, having high reflectivity and high $m$-values where $m=\gamma_{c}$ (supermirror) $/ \gamma_{c}$ (nickel) i.e. it is equal to the ratio of the critical angle of reflection, $\gamma_{c}$, of the supermirror and nickel coated optically flat glass [7]. The neutron guides with greater $m$ factor lead to increased divergence of the neutron beam which maximum value is given by the critical angle $\gamma_{c}\left[{ }^{\circ}\right]=0.1 \times m \times \lambda[\AA]$. In our preliminary feasibility analysis of the TOSCA neutron guide we have excluded the use of elliptical or more complex geometries (that are more expensive to manufacture and may 
1 considerably increase the neutron divergence at the sample position) in order to concentrate our resources on a guide with an advanced supermirror coating. Thus we have used a tapered guide in order to focus the beam at the sample position while making sure to preserve the homogeneity of the beam.

Based on extensive neutron-transport simulations and baseline studies of the guide neutronic response $[8,9,10]$, in this article we present the careful mechanical engineering design of the new TOSCA primary spectrometer and provide a review of the actual performance gains following this upgrade. A comparison between experimental observations and Monte Carlo simulations is provided, and the effect of the upgrade on the instrument spectral resolution and background have been assessed as well.

\section{The primary spectrometer}

In our design of the neutron guide, we have followed two principles: position the supermirrors to include as much of the neutron flightpath as possible, and to increase the area viewed of the water moderator by increasing the size of the shutter entrance (from its original value of $84 \mathrm{~mm} \times 84 \mathrm{~mm}$ ) while preserving the beam size at the sample position. A schematic drawing of the new $14.636 \mathrm{~m}$ long neutron guide (including $0.418 \mathrm{~m}$ of air gaps) which is dedicated to the TOSCA instrument is shown in Figure 1. The first section of the guide, G1, is installed in the new $1.937 \mathrm{~m}$ long shutter that is positioned at a distance of $1.626 \mathrm{~m}$ from the moderator centre. The shutter contains an $m=5$ straight square guide with an aperture of $100 \mathrm{~mm} \times 100 \mathrm{~mm}$. The remaining nine sections of the guide are tapered, starting from the $100 \mathrm{~mm} \times 100 \mathrm{~mm}$ entrance of section G2, all the way towards the end of section G10 with $40 \mathrm{~mm} \times 40 \mathrm{~mm}$ aperture (positioned at a distance of $16.262 \mathrm{~m}$ from the moderator centre, i.e. $0.748 \mathrm{~m}$ from the sample position). The angle of taper, $\sim 0.136494^{\circ}$, has been kept equal in each tapered section, while the $m$-factor was increased in steps from $m=5$ for sections 2 to 6, $m=6$ for sections 7 to 9 , and $m=7$ for section 10 . This gradual increase ensured optimal gain and enabled that even those short wavelength (high energy) neutrons whose divergence was sufficiently small can be retained. Geometrical parameters of each section and the neutron guide as a whole can be found in Table S1 of the supplementary information. We have chosen mostly tapered over mostly straight guide as neutron simulations pointed towards increased flux gain in the case of the former $[8,9,10]$. 
2 As part of the upgrade, the current single disc chopper [3] has been replaced by a double disc 3 chopper positioned at a distance of $9.455 \mathrm{~m}$ from the moderator centre, and between sections 4 G5 and G6 of the guide. The air gap between the two sections required to fit the discs was 5 kept to a minimum of $6.6 \mathrm{~cm}$, thus reducing the neutron flux attenuation due to scattering in 6 air. The new double disc chopper allows utilisation of all the neutron pulses arriving at 7 TOSCA, even when Target Station 1 operates in $50 \mathrm{~Hz}$ mode (albeit without access to the 8 elastic line; see reference 3 for further details about the extension of the spectral region of the 9 instrument).

11 In order to achieve the highest neutron flux at the sample position the flightpath through the 12 neutron guide is in vacuum ( $\left.\mathrm{p}=2.5 \times 10^{-2} \mathrm{mbar}\right)$. Since all the sections of the guide could not 13 be joined together in a single housing, various housings are sealed with the help of $0.5 \mathrm{~mm}$ 14 thick aluminium windows. Overall, there are a total of nine aluminium windows along the 15 beamline flightpath (i.e. between the moderator and the sample position) as indicated by the orange vertical lines in Figure 1.

Two neutron beam monitors are positioned along the flightpath; the first before the chopper, at a distance of $8.900 \mathrm{~m}$ from the moderator centre and the second after the chopper at a distance of $15.871 \mathrm{~m}$ from the moderator centre. The latter monitor is used for the normalisation of the neutron flux. The monitors allow measurements of the time-of-flight spectra of neutrons; and were made from GS20 cubes (cerium-activated lithium aluminosilicate glass, $0.25 \mathrm{~mm}$ in size) that were distributed $7 \mathrm{~mm}$ apart and across a $7 \times 6$ array $[11,12]$.

TOSCA has four ${ }^{3} \mathrm{He}$ detector tubes (two on each side of the beam) in back scattering that are used for diffraction measurements. As a result of the beam upgrade and in order to accommodate the last section of the guide they needed to be slightly moved away from the centre of the flightpath i.e. they are now positioned at an angle of $175^{\circ}$ and $176^{\circ}$ in the backward direction. Although the tubes are stationed in air, virtually all the flightpath between the sample position and the diffraction tubes is in vacuum, with the vanadium window acting as the boundary. The final section of the neutron guide, G10, is connected to the instrument by the tapered flight tube and the whole volume is kept at cryogenic vacuum $34\left(<10^{-6} \mathrm{mbar}\right)$. 
2 The instrument was not operational between the end of May 2016 and mid-February 2017.

3 By the end of November 2016, all sections of the guide were in place apart from the new

4 TOSCA shutter with the initial section of the guide within it, G1, which remained the same as

5 before the upgrade. Since the overwhelming majority of the guide was installed we tested the

6 setup for enhanced neutron flux, in order to have better idea about the influence of the guide

7 inside the shutter (installed subsequently) on the neutron flux, beam profile, spectral

8 resolution and background (see $\mathrm{SI}$ ). We will refer to this interim configuration as the $\mathrm{C} 1$

9 configuration, while the configuration before the upgrade (i.e. without the neutron guide) will

10 be denoted as C0 [13]. The last section in the shutter was installed in January 2017 to give the

11 final (so called C2) configuration. After the two weeks of commissioning measurements the

12 instrument was returned to the user programme.

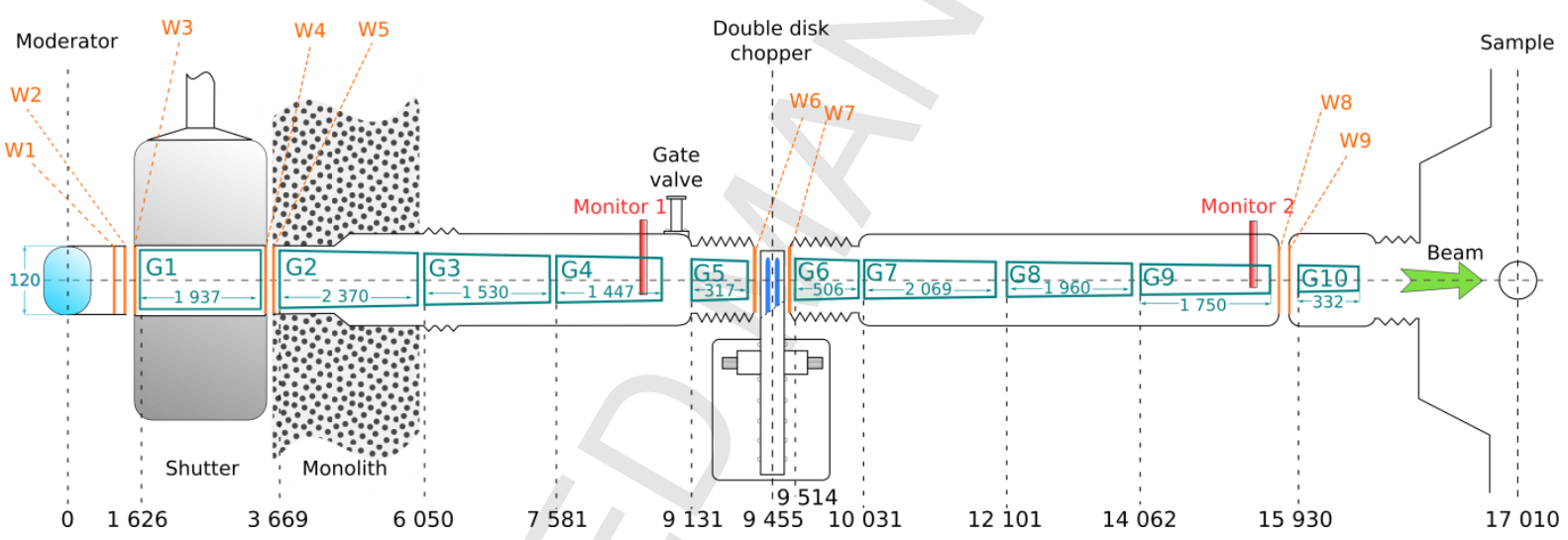

Figure 1. Schematic representation of the side view of the TOSCA neutron guide as installed on the beamline. The guide sections are numbered in the order in which they appear along the flightpath. The starting position of each section, in relation to the moderator centre, as well as its length are provided. Aluminium windows (W) are indicated by the orange vertical lines.

\section{Experimental setup}

The experimental setup used to measure the neutron flux at the sample position was described in ref. 13. The neutron sensitive component was a cuboid of cerium-doped glass scintillator, measuring $0.96 \times 0.95 \times 0.53 \mathrm{~mm}^{3}$. The TOSCA closed cycle refrigerator (CCR) was removed from its position in order to accommodate the assembly frame onto the flange, and thus the measurements at the sample position were performed in open air and at room 
1 temperature. The position of this point-sampling detector was controlled via a computer script which moved it automatically after the accumulation of 10000 frames at each spatial point $(1$ frame $=100 \mathrm{~ms}$ ), each frame containing four consecutive neutron pulses, without the need to interrupt the beam between different runs. 169 points around the beam centre (from $3.0 \mathrm{~cm}$ to $+3.0 \mathrm{~cm}$ in the $\mathrm{X}$ (horizontal) and $\mathrm{Y}$ (vertical) directions, when looking downstream) were measured, sampling the time-of-flight spectrum every $5 \mathrm{~mm}$, see Figure S1 in supplementary information (SI). Subsequently the data were calibrated to give the neutron flux at the sample position in units of neutron $\mathrm{cm}^{-2} \mathrm{~s}^{-1} \AA^{-1}$ and eventually integrated in the wavelength range of interest (see reference 13 for further details). At the time of this study, the first nine sections of the guide starting from the shutter towards the sample position were under vacuum, while section ten of the guide and the sample environment area were in air.

Computational details: The McStas software package [14] was used in order to perform Monte Carlo simulations of the TOSCA beamline. The geometrical parameters of the upgraded instrument primary beamline (see Table S1 in SI) were implemented in the virtual instrument, while the water moderator file [14] was provided by the ISIS Neutronics Group and was built using MCNP-X calculations of the actual TS1 target-reflector-moderator assembly. In the simulation the angle between the TOSCA beamline axis and the moderator face was kept at $90^{\circ}$ i.e. the moderator face and the shutter face were perfectly aligned/parallel. In reality, the beamline axis is tilted by $\sim 13.2^{\circ}$ from the line perpendicular to the moderator face [15] and this precise information has been taken into account when generating the moderator file [16]. Thus for the purposes of McStas calculations the difference between the real and the simulated angle is irrelevant, as the reality based moderator file contains all the necessary information about the neutrons travelling towards the instrument. The 'pre-guide' $\mathrm{C} 0$ configuration of the TOSCA was simulated in order to have the baseline performance of the instrument as a reference for the subsequent simulations regarding the gain in neutron flux due to the neutron guide. To calculate the performance of the supermirror neutron guide we used experimentally determined reflectivity profiles (provided by the manufacturer, Swiss Neutronics) for each section of the guide. The Mantid software package $[17,18,19,20]$ was used in order to analyse the experimental data, and details of the applied methodology of data analysis are the same as described in ref. 13. 


\section{4. Results and discussion}

3 Final (C2) configuration: The experimentally derived TOF spectrum as a function of

4 wavelength of the TOSCA neutron flux upon the guide installation (spatially averaged across 5 the $3.0 \times 3.0 \mathrm{~cm}^{2}$ surface centred around the $(0,0)$ position) is shown in Figure 2 (green trace).

6 Its features are in line with the expectations [21] since the peak in the 'moderated hump'

7 appears at around $1.1 \AA$ which is characteristic of the room temperature water moderator. Its

8 shape is characterised by the epithermal component at low wavelength and the Maxwellian

9 component that follows. Equally, in terms of its overall profile the experimental spectrum is

10 in line with the results of the Monte Carlo simulations (blue empty diamond symbols)

11 performed with the help of the McStas software, although the latter needed to be scaled down

12 by a factor of 2.53 in order to make the simulated and the experimental integrated neutron

13 flux in the region between $0.28 \AA$ and $4.65 \AA$ equal. Similar discrepancies have been observed before [13] and will be discussed in more detail later in the text. The TOSCA beam profile at the sample position is shown in Figure 3.

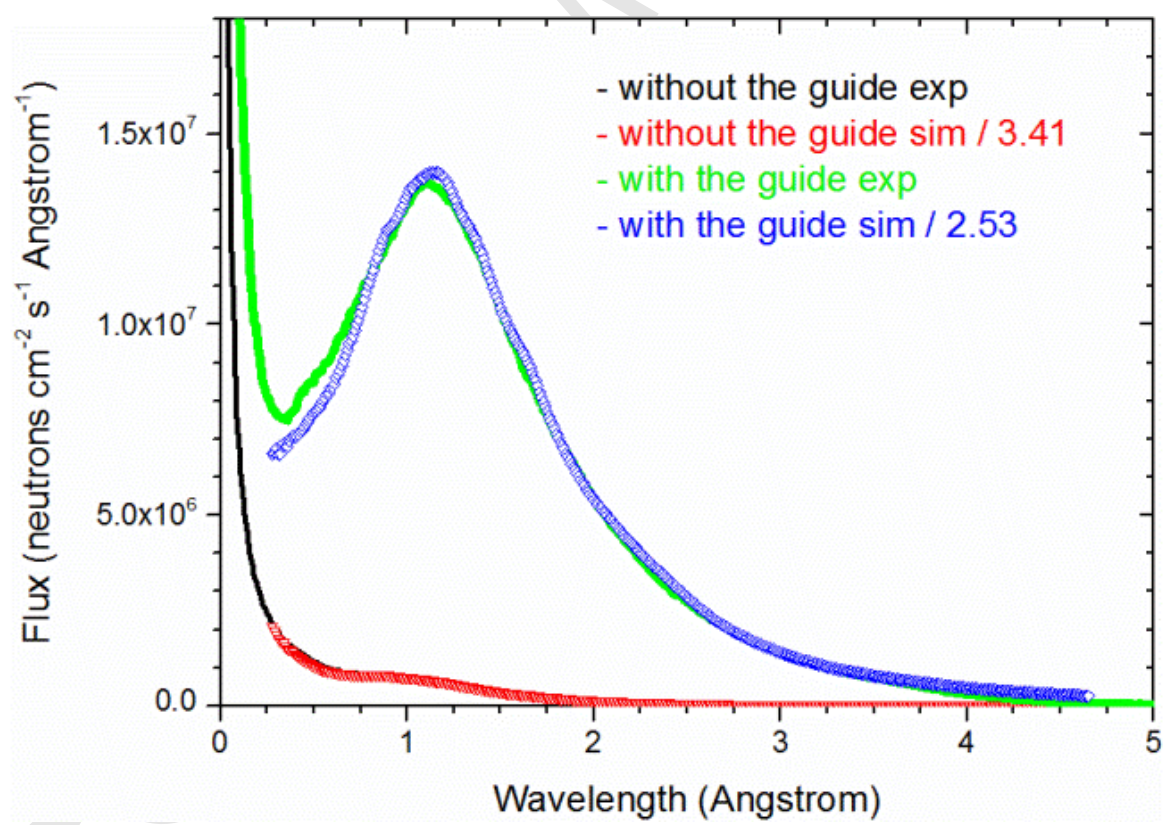

Figure 2. Neutron flux at the TOSCA sample position as a function of wavelength. Experimentally derived values (spatially averaged across the $3.0 \mathrm{x}$ $3.0 \mathrm{~cm}^{2}$ surface centred around the $(0,0)$ position) are shown by the green line, while those obtained from the Monte Carlo simulations (spatially averaged across the $4.0 \times 4.0 \mathrm{~cm}^{2}$ surface centred around the $(0,0)$ position) are shown in blue as empty diamond symbols. Monte Carlo values (not corrected for attenuation) of 
the neutron flux were scaled down by a factor of 2.53 in order to make the simulated and the experimental integrated neutron flux in the region between 0.28 $\AA$ and $4.65 \AA$ equal. For comparison, the neutron flux (experimental - black line and simulated - red empty triangle symbols) at the TOSCA sample position before the guide upgrade are also shown (configuration $\mathrm{C} 0$ ) (see reference 13 for further details).
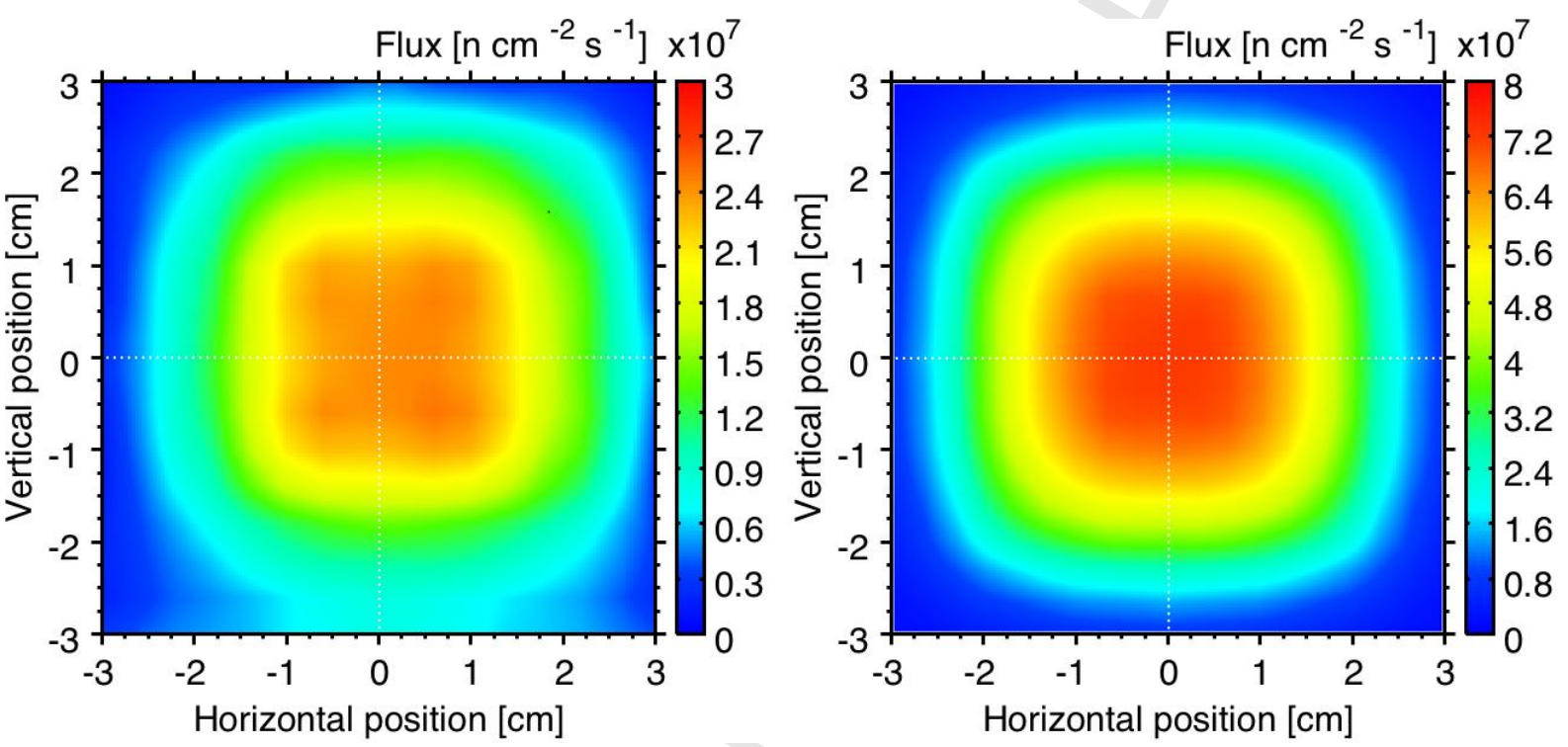

Figure 3. Measured (left) and simulated (right) neutron beam profile at the TOSCA sample position with the guide installed. The measured and calculated values of time averaged neutron flux, integrated across $0.28 \AA$ to $4.65 \AA$ wavelength range of interest to TOSCA were obtained with i.e. scaled to the average proton current-on-target of $160 \mu \mathrm{A}$, respectively.

Its central region, $2.5 \times 2.5 \mathrm{~cm}^{2}$ in area, is relatively homogeneous, while the overall beam has a roughly $4.5(\mathrm{H}) \times 4.5(\mathrm{~V}) \mathrm{cm}^{2}$ square shape (taking into account the region with the neutron flux higher than $50 \%$ of the maximum intensity) as a consequence of the neutron guide positioned along the beamline. The beam centre appears to be displaced by $\sim 2 \mathrm{~mm}$ (up and to the right when looking downstream, i.e. from the moderator towards the instrument) from the nominal primary flightpath. Such displacement (2 mm at a distance of $17010 \mathrm{~mm})$ is smaller than before the guide upgrade [13], partly as a result of the instrument repositioning, by $2 \mathrm{~mm}$ towards right i.e. the beam flightpath is now better overlapped with the sample position. The beam shift may indicate a minor shutter misalignment or possible unwanted reflections. The evaluation of the beam spatial profile as a function of wavelength is shown in 
1 Figure S3 (see SI). It appears that the beam homogeneity is preserved across neutrons with

2 various energies. In particular, the beam profile for neutrons with the wavelength between 0.8

$3 \AA$ and $1.2 \AA$ and the beam profile for neutrons with the wavelength between $2.5 \AA$ and $2.9 \AA$

4 have very similar distribution, although the latter flux is one order of magnitude smaller.

6 The McStas simulation of the TOSCA beam profile at the sample position with the guide upgrade is depicted in Figure 3. The simulated beam has a $4.0(\mathrm{H}) \times 4.0(\mathrm{~V}) \mathrm{cm}^{2}$ (taking into account the region with a neutron flux greater than $50 \%$ of the maximum intensity) Gaussian profile (due to the collimation). The flux is roughly constant across the $2.0(\mathrm{H}) \times 2.0(\mathrm{~V}) \mathrm{cm}^{2}$ surface as indicated by the horizontal cut, see Figure 4. Due to the symmetrical geometry of the square cross section guide, there are no privileged directions of reflections and, as a consequence, the results show a perfect spatial symmetry about the central position. As emphasized before, the simulated neutron flux is 2.53 times higher than the experimentally observed values. This is not surprising since the simulation was performed in vacuum while in reality, at the time of this experiment out of $17.01 \mathrm{~m}$ long flightpath between the moderator centre and the TOSCA sample position 14.1465 meters were under vacuum, while 1.3030 meters were in air, 1.5560 meters were in helium, and 0.0045 meters were in aluminium. Thus in order to compare with the experiment, the simulated values should be corrected by the factor of 0.8765 which corresponds to the transmitted neutron flux upon travel along the above described flightpath. The corrected simulated values are shown in Figure 4 as well. They are still $2.22(2.53 \times 0.8765)$ times larger than the experimental values, and the difference can be ascribed to various factors. Firstly, the muon producing target (located upstream of the TS-1 target) is not in the MCNPX model so the calculated flux should be corrected by a factor of 0.95 . Secondly, the physics model choice for MCNPX simulations can affect the flux at the level of 10-15\%. For example, if the CEM03 physics model (used in this set of simulations) is replaced by INCL4-ABLA model - the correction factor is 0.86 . Thirdly, the building of TS-1 MCNPX model [22] is still a work in progress. Recent findings [15] showed that the TS-1 target cooling water is not pure heavy water (as defined in the model) but a mixture of $80 \%$ heavy water and $20 \%$ light water, and that the $6 \mathrm{~mm}$ thick boral plate is positioned between the target and the water moderator. As a result, the calculated flux should be corrected by an additional factor of 0.87 . After applying all of these corrections, the agreement between experimental and calculated flux is well below a factor of 2. Please note that further uncertainties exist which appear to be more difficult to estimate quantitatively such as the details of material composition of the decouplers around the TS-1 moderators, 
1 precise position of the poisoning foil, the (real) vertical position of the TOSCA shutter insert, and possible operational effects [23] etc., but may explain the discrepancy.

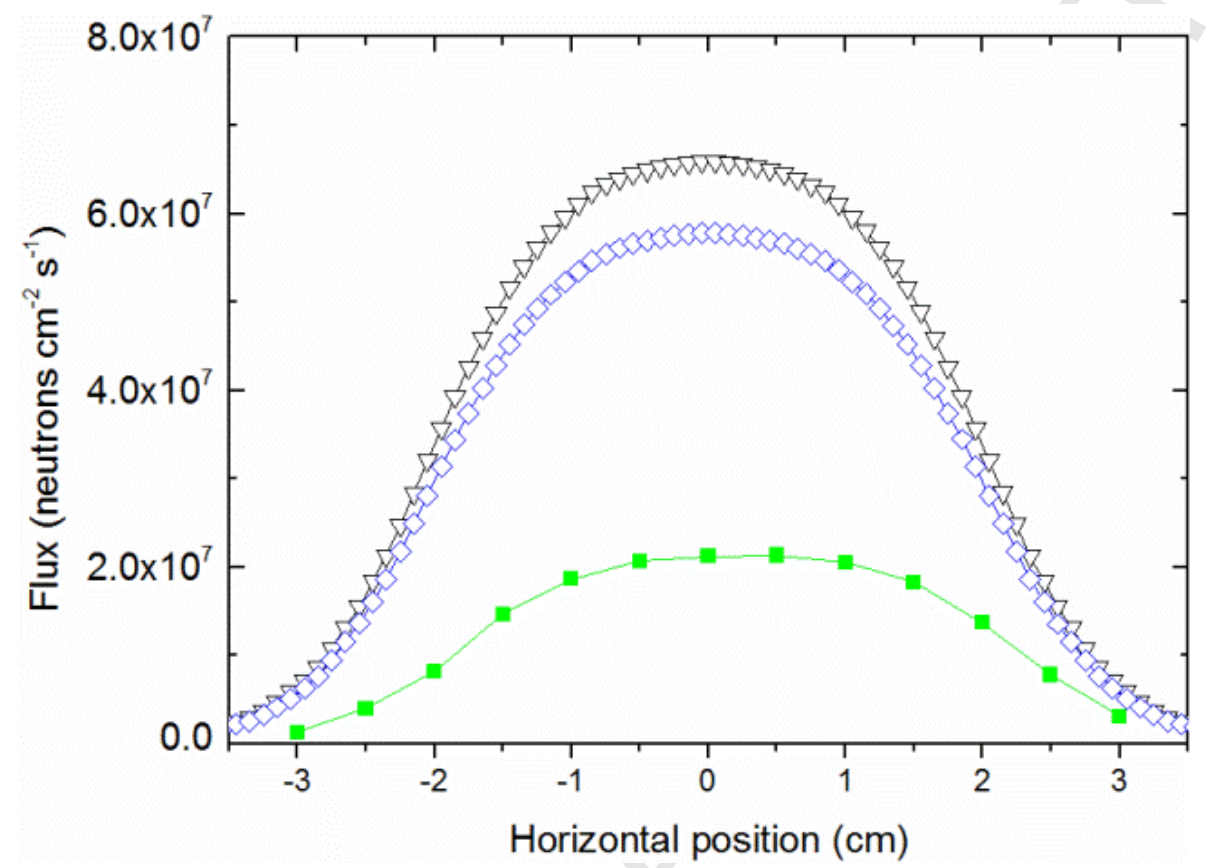

13 As can be seen from Figure 3, the measured and the calculated neutron flux at the centre of 14 the sample, the $(0,0)$ position (see SI), are $2.54 \times 10^{7}$ neutron $\mathrm{cm}^{-2} \mathrm{~s}^{-1}$ and $7.32 \times 10^{7}$ neutron

Figure 4. The experimental (green filled squares), the simulated (black empty triangles) and the corrected simulated (for attenuation in the helium, aluminium and air; blue empty diamond) neutron beam profile projected along the horizontal axis at the TOSCA sample position. The beam flux has been averaged along the vertical axis (between $-2.0 \mathrm{~cm}$ and $+2.0 \mathrm{~cm}$, i.e. within the beam height) and includes only neutrons within the wavelength range of interest to TOSCA (from $0.28 \AA$ to $4.65 \AA$ ).

$\mathrm{cm}^{-2} \mathrm{~s}^{-1}$, respectively. The corresponding values spatially averaged across $3.0 \times 3.0 \mathrm{~cm}^{2}$ and $4.0 \times 4.0 \mathrm{~cm}^{2}$ surface centred around $(0,0)$ position are $2.11 \times 10^{7}$ neutron $\mathrm{cm}^{-2} \mathrm{~s}^{-1}$ and $5.35 \mathrm{x}$ $10^{7}$ neutron $\mathrm{cm}^{-2} \mathrm{~s}^{-1}$, respectively. Between the latter two values a normalization factor of 2.53 must be applied to match the absolute values. The neutron flux values associated with the plateau shown in Figure 4, and averaged along the vertical axis (between $-2.0 \mathrm{~cm}$ and $+2.0 \mathrm{~cm}$ ) are slightly smaller. In particular the simulated values were derived with the help of the linear flux monitor, not the position sensitive detector used in the case of the beam profile shown in Figure 3. 

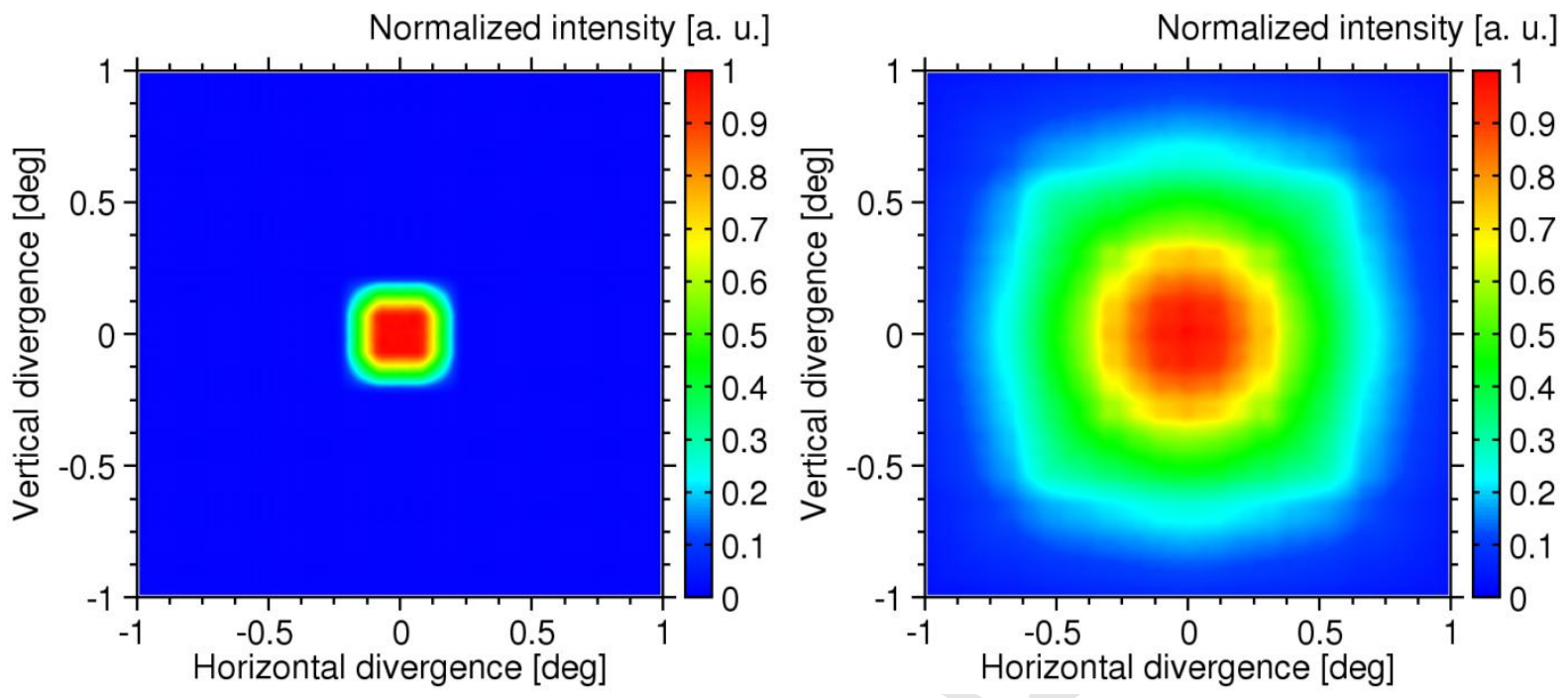

Figure 5. The TOSCA simulated divergence profile at the sample position before (left) and after (right) the guide upgrade.

6 Figure 5 shows the TOSCA simulated divergence profile at the sample position before and after the guide upgrade. In line with expectations, after the upgrade the neutrons arriving at the sample position have a larger divergence distribution: the full width at half maximum is approximately $1.3^{\circ}$, as opposed to $0.3^{\circ}$ before the upgrade. As already described, the neutron guide is able to retain neutrons (with an incident angle $<\gamma_{c}$ ) within the guide tube, rather than acting only as a simple collimator and thus by the time they reach the sample surface they can cross it at an angle larger than allowed by the direct line of sight between the moderator and the sample.

As a result of the guide being installed on TOSCA the neutron flux at the sample position has been significantly increased, see Figure 6. The green trace shows the experimentally determined gain as a function of wavelength and the black trace corresponds to the gain derived from the Monte Carlo simulations. Since measurements of the neutron flux at the sample position for the final $\mathrm{C} 2$, and before the upgrade $\mathrm{C} 0$, configurations were performed with the proton energy on target of $800 \mathrm{MeV}$ and $700 \mathrm{MeV}$, respectively, the former $\mathrm{C} 2$ neutron flux has been scaled down by a factor of 1.17 [see Figure S5 in SI] before taking the ratio to calculate the experimental gain due to the neutron guide. Additionally, as theoretical calculations were performed in vacuum, these values of the neutron flux gain need to be corrected for the attenuation as neutrons travel through helium, aluminium windows and air 
1 (blue trace) before they are compared with the experimental data. The gain in neutron flux is

2 6 times for neutrons with a wavelength of $0.5 \AA\left(2640 \mathrm{~cm}^{-1}\right), 46$ times for neutrons with a 3 wavelength of $2.5 \AA\left(105 \mathrm{~cm}^{-1}\right)$, and 82 times for neutrons with a wavelength of $4.6 \AA$ (31

$4 \mathrm{~cm}^{-1}$ ), i.e. the most significant gain is for the neutrons used to study low energy molecular 5 and lattice vibrations. The slight dip in the experimental neutron gain between $3.7 \AA$ and 4.6

$6 \AA$ is a result of the different starting times (wavelengths) at which the double disc chopper 7 started to block the slow neutrons (in order to prevent the $20 \mathrm{~ms}$ sub-frame overlap) at the 8 time of $\mathrm{C} 2(3.7 \AA)$ and $\mathrm{C} 0(4.25 \AA)$ configurations measurements. Figure S7 in the SI shows 9 the experimental (left) and simulated (right) gain in the neutron flux as a function of the 10 position within the TOSCA sample area. Since after the upgrade the neutron beam is more 11 divergent, and some neutrons can be detected at the position where there were none before, 12 the gain appears larger around the periphery than in the centre of the sample position.

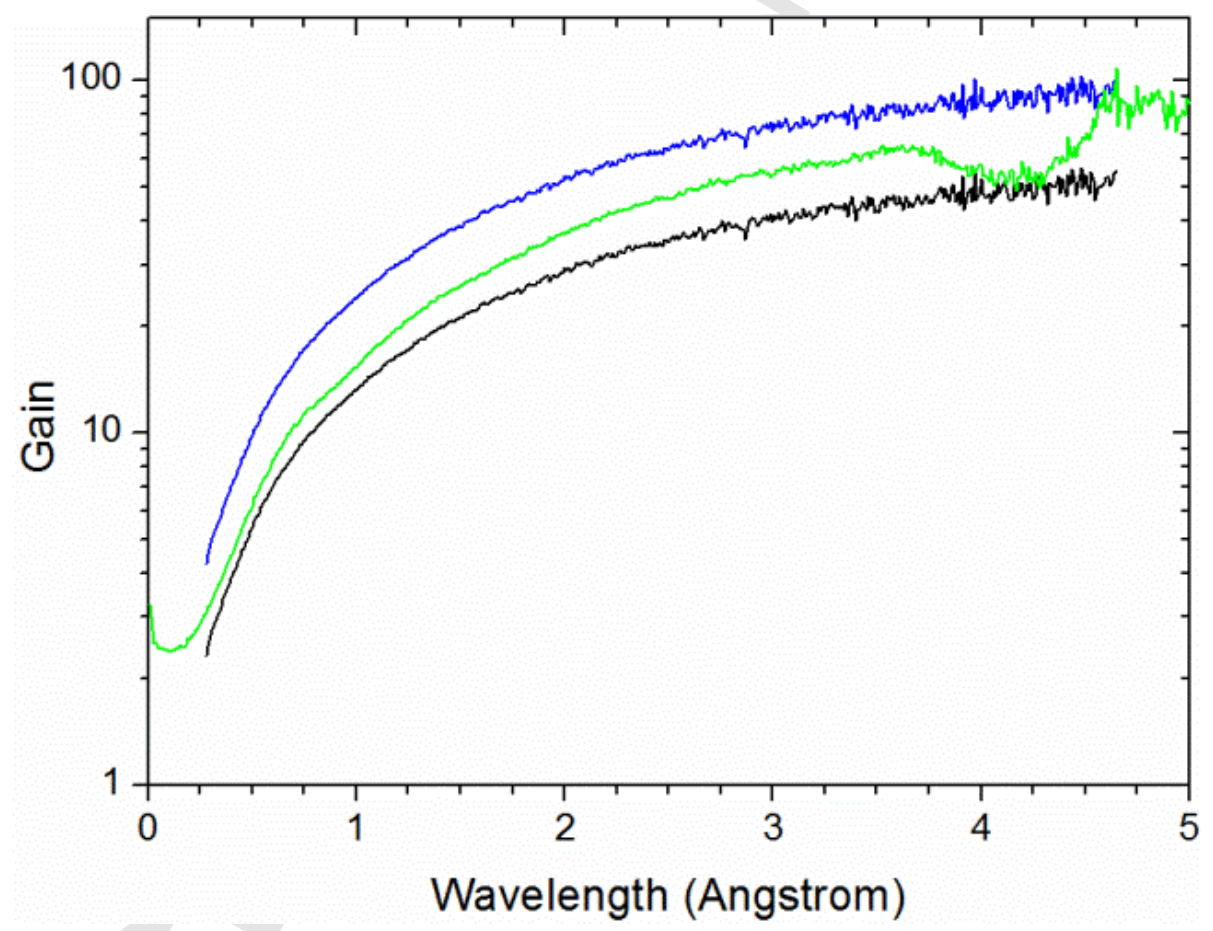

Figure 6. Gain in the neutron flux at the TOSCA sample position as a function of wavelength. Green trace shows experimental values derived with the bead detector, while black and blue traces show simulated values derived from the Monte Carlo calculations without, and with, the correction for attenuation in the helium, aluminium and air, respectively. Please see the SI for further details.

In order to understand the effect of possible shutter (i.e. initial section of the guide G1) misalignment on the neutron beam properties, we have performed additional simulations in 
which the shutter was misaligned by $0.5^{\circ}$ (to the left when looking downstream, i.e. from the moderator towards the instrument) from the intended instrument flightpath and pivoted around the shutter entrance position at a distance of $1.626 \mathrm{~m}$ from the moderator. As a consequence, the wavelength-integrated neutron flux (from $0.28 \AA$ to $4.65 \AA$ ) at the TOSCA sample position was reduced by $20 \%$ (see Figure S8 in the SI). Furthermore, it appears (see Figures S9 and S10) that the misalignment does not significantly alter the beam profile, although the beam is slightly shifted (at the sample position) in the same direction (towards left) as the misalignment. Figure S11 shows the consequence of misalignment on the beam divergence at the sample position. This suggests that as a result of the misalignment patterns in the divergence profile can be observed without alternation of the overall average 11 divergence.

In comparison to the interim $\mathrm{C} 1$ configuration (which had a simple collimation tube within the shutter and an $84 \mathrm{~mm} \times 84 \mathrm{~mm}$ entrance opening) the final C2 configuration (which has 1.937 meter long $m=5$ neutron guide inside the shutter and an enlarged $100 \mathrm{~mm} \times 100 \mathrm{~mm}$ shutter entrance) gives significantly enhanced neutron flux at the sample position; compare experimental values of the neutron flux in Figure 2 and Figure S13. For further information about the properties of the neutron beam at the TOSCA sample position in relation to $\mathrm{C} 1$ configuration please see SI. 
1 Spectral resolution and background: While the increased neutron flux at the sample position has been achieved as part of this project, it was equally important to preserve the excellent spectral resolution and low background afforded by TOSCA [3]. In order to check

4 the effect of the installed neutron guide on the instrument spectral performance we have performed neutron scattering measurements of the standard calibrant, 2,5-diiodothiophene

6 [24]. Figure 7 shows the elastic line of the 2,5-diiodothiophene sample recorded before the

7 TOSCA upgrade (black trace, $\mathrm{C} 0$ configuration) and after the upgrade (blue trace, $\mathrm{C} 2$

8 configuration). The same sample was used in both cases and the spectra were accumulated

9 for the same period of time. The spectra suggest that the spectral resolution at the elastic line 10 is essentially unchanged and below $2 \mathrm{~cm}^{-1}$.

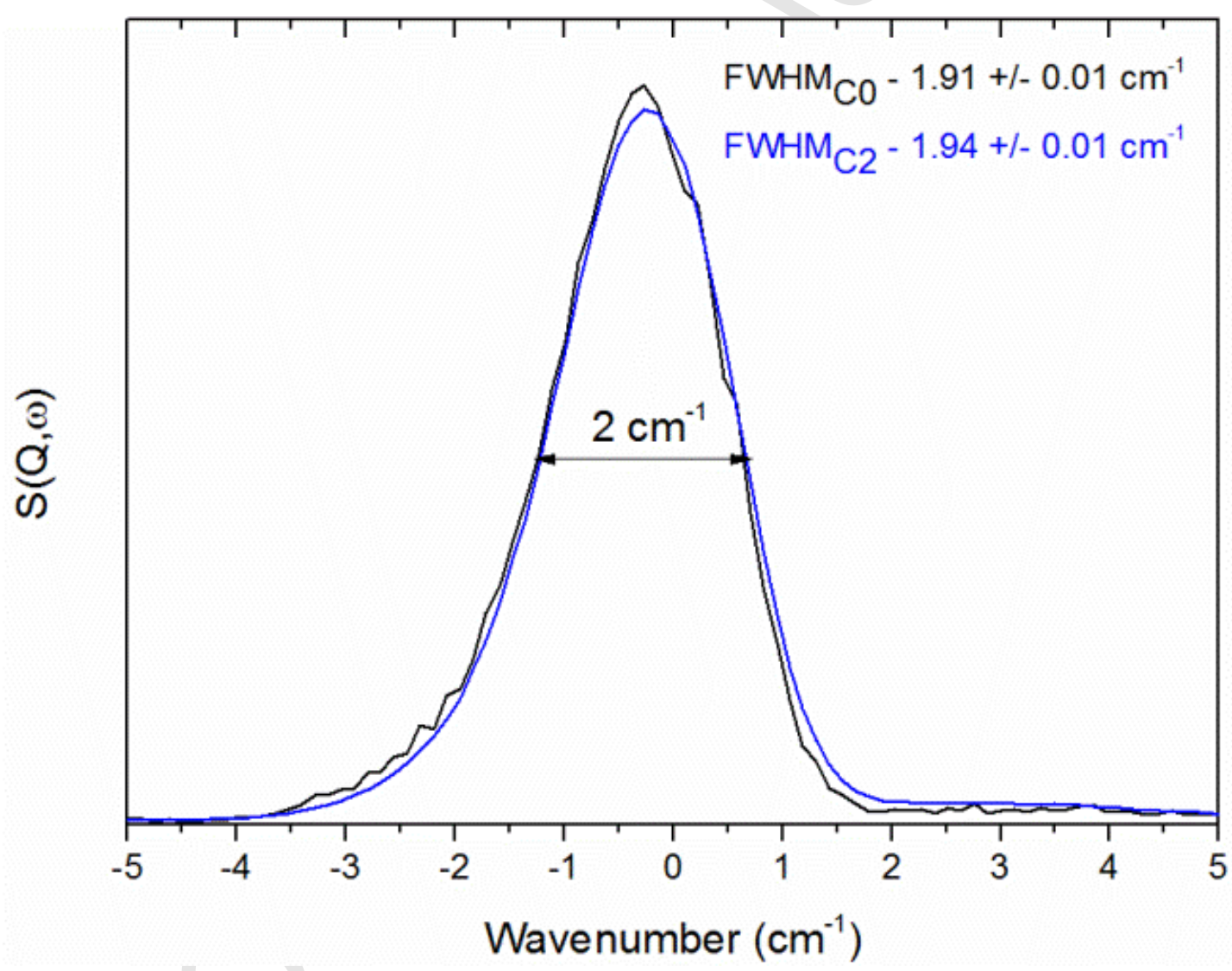

Figure 7. Elastic line of 2,5-diiodothiophene, recorded at $10 \mathrm{~K}$, before the TOSCA upgrade (black trace, C0 configuration) and after the upgrade (blue trace, C2 configuration). Note that the spectral resolution is essentially unchanged at the elastic line.

Equally, examination of the inelastic neutron scattering spectra of the same compound before and after the upgrade, see Figure 8, highlights that the spectral background has remained low 
1 across the spectral range, i.e. it has been maintained close to the pre-upgrade levels. Note that

2 the total background (black and blue trace) is not very much different from the spectral

3 background as the combined contribution of the whole instrument and the empty standard

4 TOSCA flat aluminium cell to the recorded signal (red trace) is almost negligible, and has not

5 been subtracted from the black and blue trace. Furthermore, one can observe that upon the

6 installation of the neutron guide the spectral resolution at high energy transfers is ever so

7 slightly diminished, but as the Figure S12 shows for the band associated with the $\mathrm{CH}$

8 stretches (positioned around $3085 \mathrm{~cm}^{-1}$ ) the change does not significantly alter the quality or

9 understanding of the data. In the upper right corner of Figure 8, the spectral region in between

100 and $100 \mathrm{~cm}^{-1}$ is shown on an expanded scale.

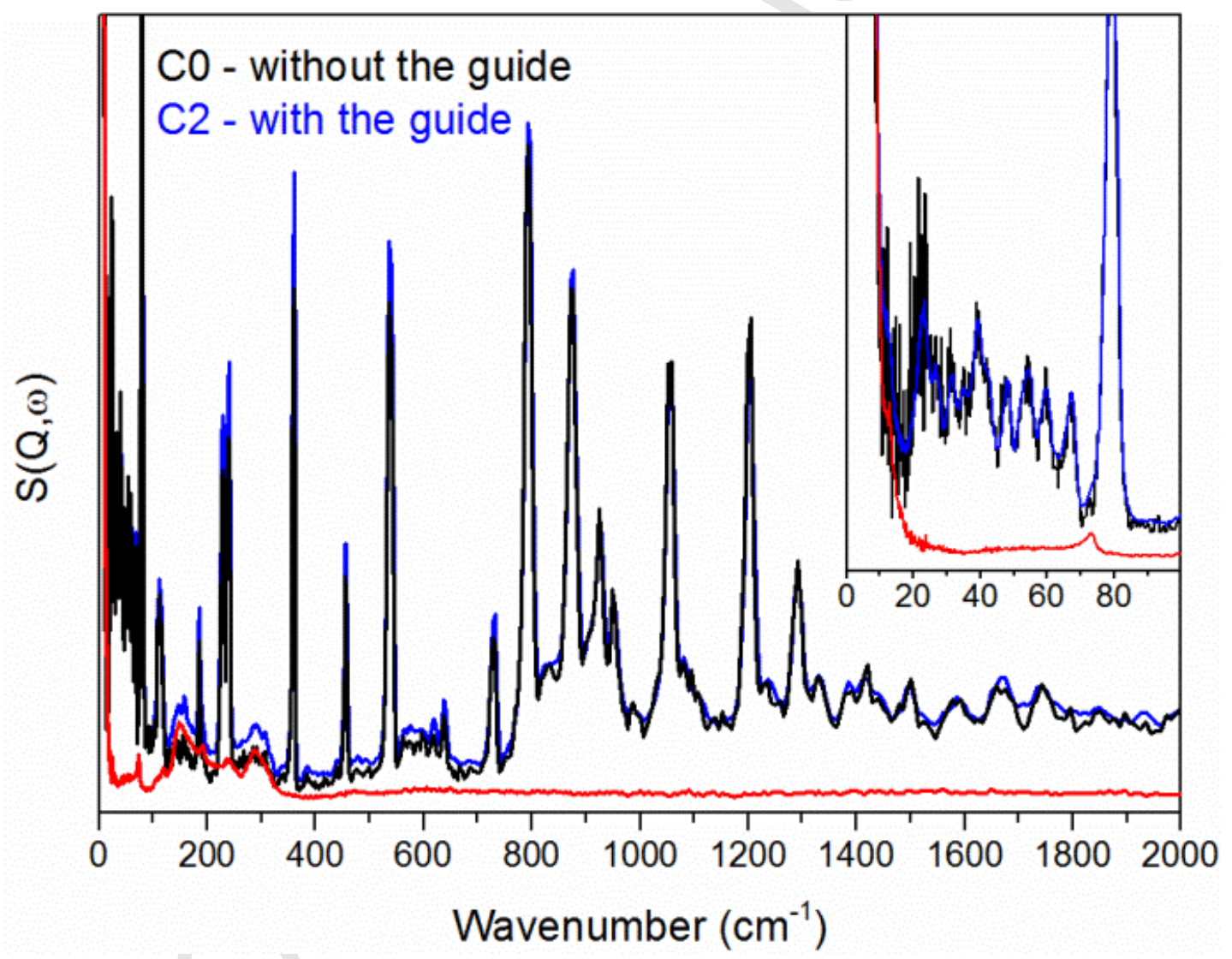

Figure 8. Inelastic neutron scattering spectra of 2,5-diiodothiophene, recorded at $10 \mathrm{~K}$, before the TOSCA upgrade (black trace, $\mathrm{C} 0$ configuration) and after the upgrade (blue trace, C2 configuration). The INS spectrum of the empty standard TOSCA flat aluminium cell (red trace - recorded after the upgrade) used to contain the sample has not been subtracted from the black or blue trace. In the upper right corner of the figure the spectral region in between 0 and $100 \mathrm{~cm}^{-1}$ is shown on an expanded scale. 


\section{5. Conclusions}

4 As part of the TOSCA primary spectrometer upgrade the simple collimator tube between the moderator and the instrument has been replaced with a supermirror guide thus leading to an increased neutron flux at the sample position. In the low energy neutron region (e.g. for neutrons with a wavelength of $4.6 \AA$ ) the gain in neutron flux of as much as 82 times has been observed. The results evidenced that the incident beam on TOSCA is reasonably square and homogeneous across $2.5(\mathrm{H}) \times 2.5(\mathrm{~V}) \mathrm{cm}^{2}$ surface, although some beam patterns can be observed, and the illuminated area is approximately $4.5(\mathrm{H}) \times 4.5(\mathrm{~V}) \mathrm{cm}^{2}$. The timeaveraged wavelength-integrated flux at the TOSCA sample centre, the $(0,0)$ position, is around $2.54 \times 10^{7}$ neutron $\mathrm{cm}^{-2} \mathrm{~s}^{-1}$ and we measured the flux as $1.38 \times 10^{7}$ neutron $\mathrm{cm}^{-2} \mathrm{~s}^{-1} \AA^{-}$ ${ }^{1}$ at the moderator peak $(\sim 1.1 \AA)$ which represents the Maxwellian component of the incident spectrum. The measurements reveal that the neutron beam profile is $\sim 27 \%$ larger (by area), and the neutron flux in the beam centre (spatially averaged across $3.0 \times 3.0 \mathrm{~cm}^{2}$ surface centred around $(0,0)$ position) is $2.53(2.22$, after correction for attenuation in the air, helium and aluminium) times smaller than predicted by the Monte Carlo calculations. The improved neutron flux allows faster measurements where useful data of hydrogen rich samples can be obtained within minutes. The upgrade will allow experiments with samples of a smaller mass that are too expensive to generate in large quantities. As a result of the upgrade, we have already noticed that the user program on TOSCA is becoming even more popular, while the number of requested days per experiment on average appears to fall. It is worthwhile noting that TOSCA capabilities could be further enhanced via upgrade of its secondary spectrometer whereby currently installed flat analysers could be exchanged for curved analysers and their surface area enlarged. Such an upgrade could potentially increase the neutron flux reaching the detectors by an order of magnitude as recent simulations suggest [25].

\section{Acknowledgements}

The authors gratefully acknowledge the Science \& Technology Facilities Council (STFC) for financial support for this project, access to the TOSCA beamline at ISIS and the use of the eScience SCARF cluster at the Rutherford Appleton Laboratory. This work has been partially 
1 research at the ISIS Facility. We thank Dr Daniele Colognesi for numerous valuable 2 discussions.

\section{References}

1. D. Colognesi, M. Celli, F. Cilloco, R.J. Newport, S.F. Parker, V. Rossi-Albertini, F.

7 Sacchetti, J. Tomkinson, M. Zoppi, TOSCA neutron spectrometer: the final configuration, 8 Appl. Phys. A - Materials Science \& Processing 74 [Suppl.] (2002) S64.

9 2. ISIS Neutron and Muon Source, TOSCA neutron spectrometer, available at 10 https://www.isis.stfc.ac.uk/Pages/tosca.aspx (accessed on the 23 of November 2017).

11 3. S.F. Parker, F. Fernandez-Alonso, A.J. Ramirez-Cuesta, J. Tomkinson, S. Rudic, R.S. 12 Pinna, G. Gorini, Recent and future developments on TOSCA at ISIS, Journal of Physics: 13 Conference Series, 554 (2014) 012003.

14 4. S.F. Parker, D. Lennon, P.W. Albers, Vibrational spectroscopy with neutrons: a review of new directions, Applied Spectroscopy, 65 (2011) 1325.

16 5. S. Rudic, A.J. Ramirez-Cuesta, S.F. Parker, F. Fernandez-Alonso, R.S. Pinna, G. Gorini,

17 C.G. Salzmann, S.E. McLain, N.T. Skipper, TOSCA international beamline review, RAL 18 Technical Reports RAL-TR-2013-015, STFC, 2013.

19 6. G.J. Kearley, V.K. Peterson (Editors) Neutron Applications in Materials for Energy, 20 Springer International Publishing Switzerland, 2015.

7. P.C.H. Mitchell, S.F. Parker, A.J. Ramirez-Cuesta, J. Tomkinson, Vibrational spectroscopy with neutrons - with applications in chemistry, biology, materials science and catalysis, World Scientific Publishing Co. Pte. Ltd., Singapore, 2005.

8. J. Fernandez Castanon, Monte-Carlo simulations for the development of TOSCA's guide at ISIS, RAL Technical Reports RAL-TR-2013-002, STFC, 2013.

9. R.S. Pinna, Monte Carlo simulations of the TOSCA neutron spectrometer: assessment of current performance and future upgrades, MSc Thesis, University of Milano-Bicocca, 2014. 10. R.S. Pinna, S. Rudic, S.F. Parker, G. Gorini, F. Fernandez-Alonso, Monte Carlo simulations of the TOSCA spectrometer: assessment of current performance and future upgrades, EPJ Web of Conferences, 83 (2015) 03013.

31 11. ISIS Neutron Beam Monitor, Quantum Detectors Ltd., Harwell, Oxford, UK, available at http://quantumdetectors.com/neutrons (accessed on the 23 of November 2017). 
1 12. 6-Lithium Glass - bespoke to your application, Scintacor, Cambridge, UK, available at 2 https://scintacor.com/products/6-lithium-glass/ (accessed on the 23rd of November 2017).

3 13. R.S. Pinna, S. Rudic, M.J. Capstick, D.J. McPhail, D.E. Pooley, G.D. Howells, G. Gorini,

4 F. Fernandez-Alonso, Detailed characterisation of the incident neutron beam on the TOSCA

5 spectrometer, Nuclear Inst. and Methods in Physics Research, A 870 (2017) 79.

6 14. P. Willendrup, E. Farhi, K. Lefmann, McStas 1.7 - a new version of the flexible Monte

7 Carlo neutron scattering package, Physica B, 350 (2004) e735-e737.

8 15. G. Skoro, S. Lilley, R. Bewley, Neutronics analysis of target, moderators and reflector 9 design for the ISIS TS-1 project, Physica B: Condensed Matter, https://doi.org/10.1016/j.physb.2017.12.060

16. S. Ansell and D. Champion, The ISIS_moderator component, McStas simulation package,

http://www.mcstas.org/download/components/contrib/ISIS_moderator.html

13 (accessed on the 23rd of November 2017).

14 17. Mantid (2013): Manipulation and analysis toolkit for instrument data. Mantid Project. 15 https://www.mantidproject.org/Main_Page (accessed on the 23 of November 2017). 18. O. Arnold, J.C. Bilheux, J.M. Borreguero, A. Buts, S.I. Campbell, L. Chapon, M. Doucet, N. Draper, R. Ferraz Leal, M.A. Gigg, V.E. Lynch, A. Markvardsen, D.J. Mikkelson, R.L. Mikkelson, R. Miller, K. Palmen, P. Parker, G. Passos, T.G. Perring, P.F. Peterson, S. Ren, M.A. Reuter, A.T. Savici, J.W. Taylor, R.J. Taylor, R. Tolchenov, W. Zhou, J. Zikovsky, Mantid - Data analysis and visualization package for neutron scattering and $\mu$ SR experiments, Numerical Instruments and Methods in Physical Research A 764 (2014) 156 166.

19. E. Oram, An overview of the development of indirect inelastic data reduction and analysis in MANTID between July 2015-July 2016, RAL Technical Reports RAL-TR-201611, STFC, 2016.

20. L. McCann, Overview of developments in MANTID relating to indirect inelastic spectroscopy July 2016-July 2017, RAL Technical Reports RAL-TR-2017-12, STFC, 2017. 21. C.G. Windsor, Pulsed Neutron Scattering, Taylor \& Francis Ltd, London, 1981. 22. G.P. Škoro and S. Ansell, Neutronics modelling for the ISIS TS-1 upgrade, Proceedings of the 21st Meeting of the International Collaboration on Advanced Neutron Sources ICANS XXI, Japan Atomic Energy Agency, 2016, pp141-147. 
1 24. S.F. Parker, J.L. Parker, M. Jura, Structure and vibrational spectra of 2,5-diiodothiophene:

2 a model for polythiophene, Journal of Physical Chemistry C, 121 (2017) 12636-12642.

3 25. R.S. Pinna, S. Rudic, M. Zanetti, D. Zacek, S.F. Parker, G. Gorini, F. Fernandez-Alonso,

4 Monte Carlo simulations for the TOSCA secondary spectrometer upgrade, RAL Technical 5 Reports RAL-TR-2017-013, STFC, 2017. 